\title{
Stability Analysis of Wireless Coupled-Oscillator Circuits
}

\author{
Mabel Pontón, Almudena Suárez \\ University of Cantabria, Santander, Spain
}

\begin{abstract}
Distributed synchronization of sensor networks can be achieved by coupling the oscillator signals of the sensor nodes. Previous works describe the coupling effects in an idealized manner, with constant scalar coefficients. Here a realistic analysis of the coupled-system dynamics is presented for the first time to our knowledge, taking into account the antenna gains and propagation effects on the amplitude and phase values of the equivalent current sources, injecting the oscillator elements. The new formulation provides the synchronized oscillation frequency and amplitude and phase distributions of the coupled system. Distinct oscillation modes, with different phase shifts between the oscillator elements, are identified, associated with the system symmetry. The stability properties of these modes change with the distance between the oscillator elements. The possibility to impose in-phase operation by tuning of the oscillator elements is demonstrated. Good agreement is obtained between simulation and measurements.
\end{abstract}

Index Terms - Wireless locking, coupled oscillators, stability.

\section{INTRODUCTION}

A common time scale between the nodes of a sensor network [1] enables applications such as cooperative transmissions, data-fusion of time-sensitive measurements or moving object tracking. The synchronization can be obtained through the broadcast of a beacon timing signal from a central node. However, in fully distributed scenarios, this broadcast will not be possible, and the common time scale can only be achieved through a distributed synchronization. This can be done through exchange of packets carrying time stamps [1], which suffer from random delays in the construction and processing of the packet. Alternative methods based on the wireless coupling of the oscillators in the sensor nodes [1]-[3] have been proposed. In pulse-coupled oscillators, pulsed signals are transmitted and the nodes must detect the time of arrival of the pulses [1]-[2], which is demanding in terms of frequency bandwidth. In analog coupling, each node transmits a signal proportional to its local oscillation, with all the nodes transmitting and receiving continuously at the same time [1]-[3].

Although there are several previous works on wireless coupled oscillators, they rely on ideal models [2]-[3] of both the oscillator circuits, described in terms of phase variables only, and the coupling action, reduced to constant scalar coefficients. Here an in-depth investigation of the dynamics of the wireless coupled system is carried out, considering realistic oscillator models [4]-[7] and a detailed description of the coupling effects resulting from the antenna gain and signal propagation. These coupling effects are distance and frequency dependent, and will have a significant impact on the synchronized oscillation frequency, phase distribution and stability properties. Distinct oscillation modes, associated with the system symmetries, will be theoretically identified and their stability properties will be shown to depend on the distance between the oscillator elements.

\section{WIRELESS-COUPLED OSCILLATOR SYSTEM}

Under weak coupling conditions, the oscillators, assumed to be slightly different, can be modeled with the derivatives of their admittance function (of zero value at the free-running oscillator) about their free-running solutions, calculated through finite differences. This is done with the aid of an auxiliary generator $(\mathrm{AG})$ in harmonic balance (HB) [4]-[7]. For simplicity, a system of three oscillators will be considered [Fig. 1(a)], although the formulation can be extended to any number $M$.
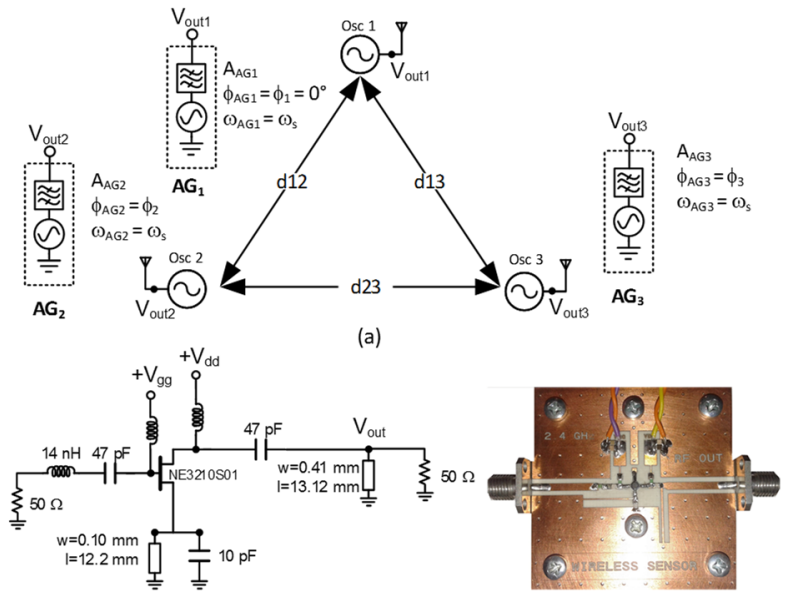

(b)

(c)

Fig. 1 Wireless coupled oscillator system. (a) Schematic. (b) Oscillator design based on the FET NE3210S01 (c) Prototype built on Rogers 4003C. The connection of the three AGs used for the derivative calculation and in the circuit-level analysis is also represented.

Prior to the coupling, the free-running oscillators exhibit the amplitudes and frequencies $V_{m o}, \omega_{m o}$, where $m=1$ to 3 . The wireless-coupled system is governed by the equations:

$$
\begin{aligned}
& Y_{1 v} \Delta V_{1}+Y_{1 \omega} \Delta \omega=\gamma_{21} A_{12} e^{-j \alpha_{12}} e^{j \phi_{2}}+\gamma_{31} A_{13} e^{-j \alpha_{13}} e^{j \phi_{3}} \\
& +Y_{1 \omega} \Delta \omega_{21} \\
& Y_{2 v} \Delta V_{2}+Y_{2 \omega} \Delta \omega=\gamma_{21}^{-1} A_{12} e^{-j \alpha_{12}} e^{-j \phi_{2}}+\gamma_{32} A_{23} e^{-j \alpha_{23}} e^{j\left(\phi_{3}-\phi_{2}\right)} \\
& Y_{3 v} \Delta V_{2}+Y_{3 \omega} \Delta \omega=\gamma_{31}^{-1} A_{13} e^{-j \alpha_{13}} e^{-j \phi_{3}}+\gamma_{32}^{-1} A_{23} e^{-j \alpha_{23}} e^{j\left(\phi_{2}-\phi_{3}\right)} \\
& +Y_{3 \omega} \Delta \omega_{23}
\end{aligned}
$$

where $\Delta V_{m}=V_{m}-V_{m o}$ are the amplitude increments, $\Delta \omega=\omega-\omega_{m o}, \phi_{2}$ and $\phi_{3}$ are the phase values of the second and third elements, $\phi_{1}=0$ is the phase origin and 
$\gamma_{m k}=V_{m o} / V_{k o}$ where $m=1$ to 3 . The constant offset frequencies $\Delta \omega_{21}=\omega_{20}-\omega_{10}, \Delta \omega_{23}=\omega_{20}-\omega_{30}$ reduce the sensitivity to the injection signals and should be minimized by making the free-running frequencies as similar as possible. The terms due to the propagation effects [Fig. 1(a)] are:

$$
\begin{aligned}
& A_{m k}\left(\omega, d_{m k}\right)=\left(2 \sqrt{G_{t, m} G_{r, k} / L_{m k}}\right) / R \\
& L_{m k}=\left(2 \omega d_{m k} / c\right)^{2} ; \alpha_{m k}\left(\omega, d_{m k}\right)=\omega d_{m k} / c
\end{aligned}
$$

where $L_{m k}$ is the basic propagation loss. For each set of distances, $d_{12}, d_{23}, d_{13}$, the system unknowns are $\Delta V_{m}, \phi_{2}, \phi_{3}$ and $\omega$. In the wireless-locking case, the coupling coefficients have significant frequency dependence (both in magnitude and phase), unlike the usual coupled oscillators [5]-[6]. To investigate the distinct oscillation modes, system (1) will be particularized to equal oscillator elements and equal distances $d$, which provides:

$$
\left\{\left[\begin{array}{ccc}
Y(V, \omega) & 0 & 0 \\
0 & Y(V, \omega) & 0 \\
0 & 0 & Y(V, \omega)
\end{array}\right]-\left[\begin{array}{ccc}
0 & A e^{-j \alpha} & A e^{-j \alpha} \\
A e^{-j \alpha} & 0 & A e^{-j \alpha} \\
A e^{-j \alpha} & A e^{-j \alpha} & 0
\end{array}\right]\right\}\left[\begin{array}{c}
\bar{V}_{1} \\
\bar{V}_{2} \\
\bar{V}_{3}
\end{array}\right]=0
$$

where $Y(V, \omega)=Y_{v} \Delta V+Y_{\omega} \Delta \omega$ and $\bar{V}_{m}=V_{o} e^{j \phi_{m}}$. The modes are associated with the eigenvalues and eigenvectors of the coupling circular matrix, after the minus sign, rewritten as:

$$
\left[\begin{array}{ccc}
0 & A e^{-j \alpha} & A e^{-j \alpha} \\
A e^{-j \alpha} & 0 & A e^{-j \alpha} \\
A e^{-j \alpha} & A e^{-j \alpha} & 0
\end{array}\right]=\left[\begin{array}{ccc}
C_{11} & C_{12} & C_{13} \\
C_{13} & C_{11} & C_{12} \\
C_{12} & C_{13} & C_{11}
\end{array}\right]
$$

The corresponding eigenvalues have the general form [8],[9]:

$$
\lambda_{n}=C_{11}+C_{12} e^{j \frac{n 2 \pi}{3}}+C_{13} e^{j \frac{2 n 2 \pi}{3}}=A e^{-j \alpha} e^{j \frac{n 2 \pi}{3}}+A e^{-j \alpha} e^{j \frac{2 n 2 \pi}{3}}
$$

where $n=0$ to 2 , and the respective eigenvectors are:

$$
\bar{V}_{n}=\left(V_{o} / \sqrt{3}\right)[1 \quad \exp (j n 2 \pi / 3) \quad \exp (j 2 n 2 \pi / 3)]^{T}
$$

The two distinct eigenvalues are:

$$
\lambda_{0}=2 A e^{-j \alpha}, \quad \lambda_{1}=\lambda_{2}=-A e^{-j \alpha}
$$

which, taking (6) into account, correspond to solutions with $0^{\circ}$ and $120^{\circ}$ phase shift between the oscillator elements, respectively. When exciting the oscillator nodes with a voltage vector agreeing with the eigenvector $\bar{V}_{n}$, all the oscillator nodes will "see" the same admittance function , $\lambda_{n}$, when looking into the coupling network. Thus, the oscillation condition corresponding to the $0^{\circ}$ mode is:

$$
\begin{aligned}
& G_{v}\left(V_{1}-V_{o}\right)+G_{\omega}\left(\omega-\omega_{o}\right)=2 c G_{a} \cos (\omega d / c) /(\omega R d) \\
& B_{v}\left(V_{1}-V_{o}\right)+B_{\omega}\left(\omega-\omega_{o}\right)=-2 c G_{a} \sin (\omega d / c) /(\omega R d)
\end{aligned}
$$

where expressions of $A$ and $\alpha$ in (2) have been replaced and $G_{a}=\sqrt{G_{t} G_{r}}$. The oscillation condition for the $120^{\circ}$ mode is:

$$
\begin{aligned}
& G_{v}\left(V_{1}-V_{o}\right)+G_{\omega}\left(\omega-\omega_{o}\right)=-c G_{a} \cos (\omega d / c) /(\omega R d) \\
& B_{v}\left(V_{1}-V_{o}\right)+B_{\omega}\left(\omega-\omega_{o}\right)=c G_{a} \sin (\omega d / c) /(\omega R d)
\end{aligned}
$$

Results of the general system (1) with equidistant nodes will approach (8) and (9) as the oscillator designs become more similar. Solving for $\omega$ in the two cases:

$$
\begin{aligned}
& \omega-\omega_{o}=\frac{-2 c G_{a}\left[G_{v} \sin (\omega d / c)+B_{v} \cos (\omega d / c)\right]}{(\omega R d)\left(G_{v} B_{\omega}-G_{\omega} B_{v}\right)}, 0^{\circ} \\
& \omega-\omega_{o}=\frac{c G_{a}\left[G_{v} \sin (\omega d / c)+B_{v} \cos (\omega d / c)\right]}{(\omega R d)\left(G_{v} B_{\omega}-G_{\omega} B_{v}\right)}, 120^{\circ}
\end{aligned}
$$

Comparing the two expressions, the coupling strength is weaker for $120^{\circ}$ and the frequency increments have opposite signs in the two modes. The magnitude of $\omega-\omega_{0}$ decreases with $d$, due to a weaker coupling. For a sufficiently large $d$, the oscillators will tend to their original free-running frequency and hence to a non-synchronized regime. However, for a large $d$, the oscillators could be kept synchronized increasing the antenna gains. The method has been applied to an oscillator system based on the FET NE3210S01 [Fig. 1(b)] at 2.4 GHz. Fig. 2 shows the variation of $\omega$ versus $d$ predicted with (10). Only the solution with $0^{\circ}$ exhibits turning points, which is consistent with the stronger coupling for this oscillation mode. Results are compared with costly circuit-level HB simulations using three AGs. However, the HB simulations are unable to complete the solution curves.

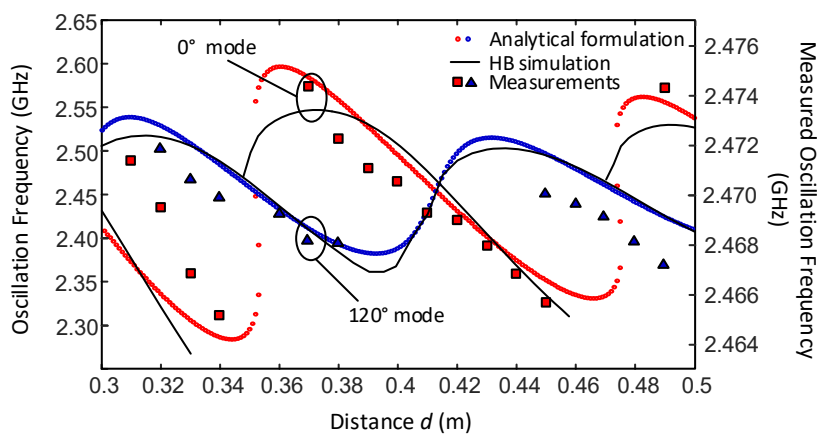

Fig. 2 Variation of the oscillation frequency versus the distance $d$ for the two oscillation modes. Measurements are superimposed.

For simplicity, a wireless coupled oscillators system of $N=3$ oscillators have been considered. However, the same analysis procedure can be applied for a generalized system of 
$N$ oscillators, in a ring topology, just increasing the dimension of system (3) to $N$ and calculating the corresponding eigenvalues and eigenvectors associated to the $N$ x $N$ coupling circular matrix. Note that the extended system is formally identical to (3).

\section{StABILITY ANALYSIS}

The stability analysis is applied to the general system (1), since the symmetric configuration is a particular case of (1). A small perturbation is introduced, which gives rise to the time varying increments $\delta V_{m}(t), \delta \phi_{m}(t)$ in the synchronized oscillator amplitude and phase of the form $\left[V_{m o}+\delta V_{m}(t)\right] \exp \left[-j \phi_{m}+\delta \phi_{m}(t)\right]$. The expression for the perturbation frequency is the same derived in [10]-[11], which can be obtained from the time derivative of the complex envelope of the voltage at the observation node $V_{m}(t) \exp \left(-j \phi_{m}\right)$, taking the original complex waveform as common factor:

$$
\dot{V}_{m}(t)=\left[-\frac{j \delta \dot{V}_{m}(t)}{V_{m o}+\delta V_{m}(t)}+\delta \dot{\phi}_{m}(t)\right] V_{m}(t) e^{j \phi_{m}(t)}
$$

Considering $\delta V_{m}(t) \rightarrow 0$ in (11) the complex frequency increment $s$ can be expressed as $-j \delta \dot{V}_{m}(t) / V_{m o}+\delta \dot{\phi}_{m}(t)$.

In matrix form, the perturbed coupled system is:

$$
\begin{aligned}
& {\left[\begin{array}{cccccc}
B_{1 \omega} / V_{1} & A_{\omega, 12} & A_{\omega, 13} & G_{1 \omega} & A_{\omega, 15} & A_{\omega, 16} \\
-G_{1 \omega} / V_{1} & A_{\omega, 22} & A_{\omega, 23} & B_{1 \omega} & A_{\omega, 25} & A_{\omega, 26} \\
A_{\omega, 31} & B_{2 \omega} / V_{2} & A_{\omega, 33} & A_{\omega, 34} & G_{2 \omega} & A_{\omega, 36} \\
A_{\omega, 41} & -G_{2 \omega} / V_{2} & A_{\omega, 43} & A_{\omega, 44} & B_{2 \omega} & A_{\omega, 46} \\
A_{\omega, 51} & A_{\omega, 52} & B_{3 \omega} / V_{3} & A_{\omega, 54} & A_{\omega, 55} & G_{3 \omega} \\
A_{\omega, 61} & A_{\omega, 62} & -G_{3 \omega} / V_{3} & A_{\omega, 64} & A_{\omega, 65} & B_{3 \omega}
\end{array}\right]\left[\begin{array}{l}
\delta \dot{V}_{1} \\
\delta \dot{V}_{2} \\
\delta \dot{V}_{3} \\
\delta \dot{\phi}_{1} \\
\delta \dot{\phi}_{2} \\
\delta \dot{\phi}_{3}
\end{array}\right]=} \\
& {\left[\begin{array}{cccccc}
-G_{1 v} & 0 & 0 & S_{21}+S_{31} & -S_{21} & -S_{31} \\
-B_{1 v} & 0 & 0 & -\left(C_{21}+C_{31}\right) & C_{21} & C_{31} \\
0 & -G_{2 v} & 0 & -S_{12} & S_{12}+S_{32} & -S_{32} \\
0 & -B_{2 v} & 0 & C_{12} & -\left(C_{12}+C_{32}\right) & C_{32} \\
0 & 0 & -G_{3 v} & -S_{13} & -S_{23} & S_{13}+S_{23} \\
0 & 0 & -B_{3 v} & C_{13} & C_{23} & -\left(C_{13}+C_{23}\right)
\end{array}\right]\left[\begin{array}{l}
\delta V_{1} \\
\delta V_{2} \\
\delta V_{3} \\
\delta \phi_{1} \\
\delta \phi_{2} \\
\delta \phi_{3}
\end{array}\right]}
\end{aligned}
$$

where $A_{\omega, i j}$ are terms directly obtained from the frequency differentiation of the coupling coefficients in (2), and $S_{k m}=A_{m k} \sin \left(\phi_{k}-\phi_{m}-\alpha_{m k}\right), C_{k m}=A_{m k} \cos \left(\phi_{k}-\phi_{m}-\alpha_{m k}\right)$. Fig. 3 compares the poles resulting from (12) with those obtained with a costly pole-zero identification at circuit level [12], for the $0^{\circ}$ and $120^{\circ}$ modes. The real part of the poles is traced versus $d$. In applications requiring an in-phase operation, one should choose $d$ values for which only the $0^{\circ}$ mode is stable. Due to the element tolerances, the oscillator admittance functions will not be identical, so the solution will deviate from the in-phase one. However, and provided that the oscillator discrepancies are not too big, it will be possible to impose the inphase solution, through oscillator tuning. Setting the phase variables to zero in (1) and introducing the tuning voltages
$\Delta \eta_{1}, \Delta \eta_{3}$ in the two outermost oscillators, one obtains:

$$
\begin{aligned}
& Y_{1 v} \Delta V_{1}+Y_{1 \omega} \Delta \omega+Y_{1 \eta} \Delta \eta_{1}=\gamma_{21} A e^{-j \alpha}+\gamma_{31} A e^{-j \alpha} \\
& Y_{2 v} \Delta V_{2}+Y_{2 \omega} \Delta \omega=\gamma_{21}^{-1} A e^{-j \alpha}+\gamma_{32} A e^{-j \alpha} \\
& Y_{3 v} \Delta V_{2}+Y_{3 \omega} \Delta \omega+Y_{3 \eta} \Delta \eta_{3}=\gamma_{31}^{-1} A e^{-j \alpha}+\gamma_{32}^{-1} A^{-j \alpha}
\end{aligned}
$$

where very similar free-running frequencies and equidistant oscillators are assumed. Solving (13) in the unknowns $\Delta V_{k}, \Delta \omega, \Delta \eta_{1}, \Delta \eta_{3}$, one obtains the $0^{\circ}$ mode. However, a mode with phase shifts about $120^{\circ}$ will also coexist, as shown later. The stability of the solution obtained for $d=0.42 \mathrm{~m}$ has been analyzed with (12) and with pole-zero identification at circuit level [Fig. 4(a)]. The poles are shown in Fig. 4(b). The measured waveforms, after slight tuning of the gate bias, are shown in Fig. 5(a) and (b), for $d=0.4 \mathrm{~m}$ and $d=0.35 \mathrm{~m}$, respectively. Because the oscillators are not very different, the two modes vary versus $d$ in a manner similar to Fig. 2, where measurements are superimposed. At $d=0.38 \mathrm{~m}$ the two modes are stable, which has been confirmed experimentally [Fig. 5(c)]. In agreement with the theoretical predictions, the $0^{\circ}$ mode has a higher synchronization frequency than the $120^{\circ}$ mode. Note that there are relatively large $d$ intervals in which only one of the two modes is stable.
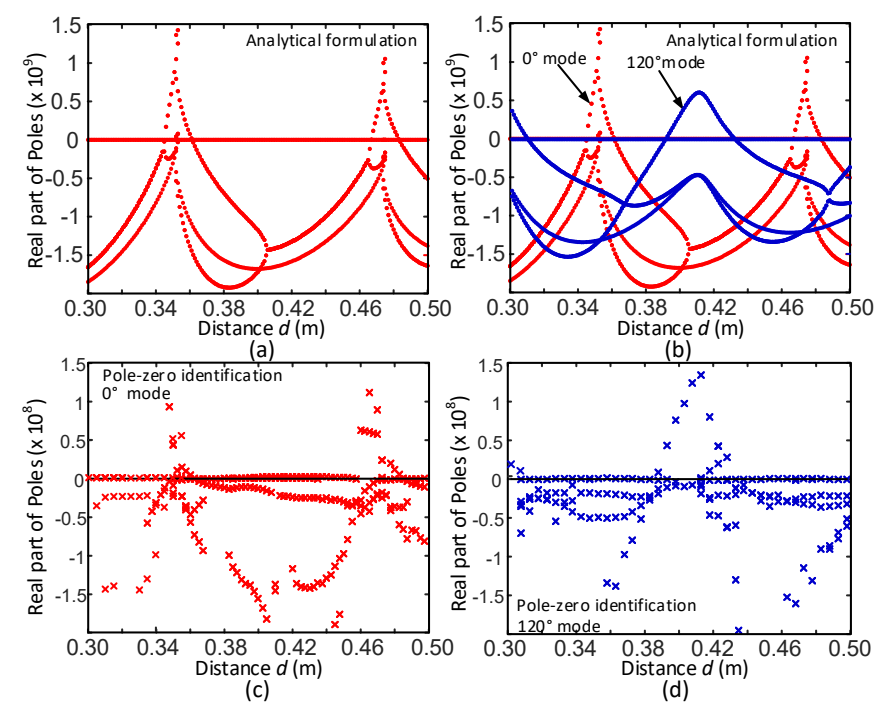

Fig. 3 Comparison of the poles resulting from (12) and the ones obtained with pole-zero identification [11]. (a) and (c) In-phase mode. (b) and (d) $120^{\circ}$.
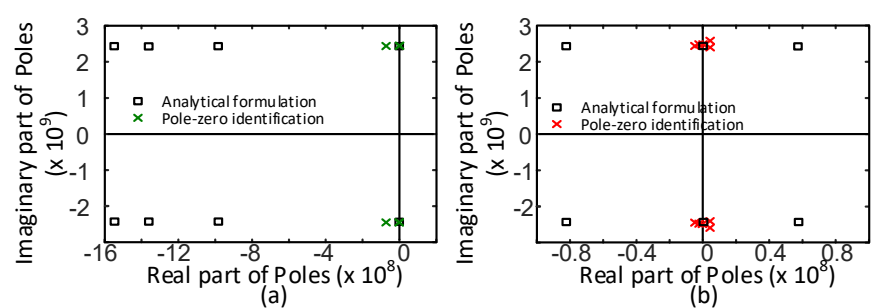

Fig. 4 Stability analysis for $d=0.42 \mathrm{~m}$ after using (13) to obtain $0^{\circ}$ phase shift. Results obtained with (12) are compared with pole-zero identification. (a) $0^{\circ}$ mode. (b) $120^{\circ}$ mode. 


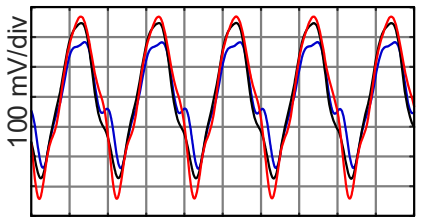

(a)

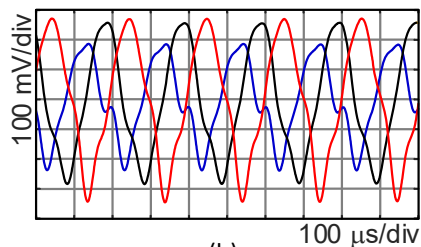

(b)

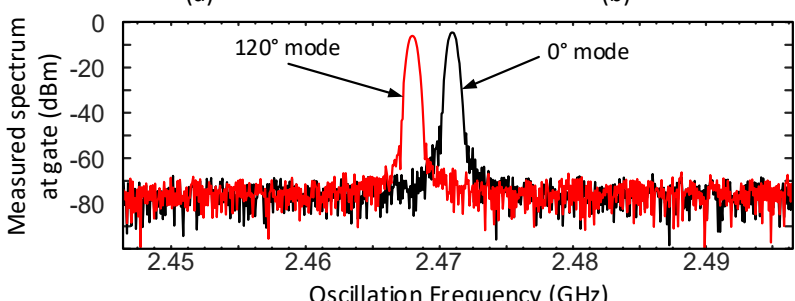

(c)

Fig. 5 Experimental results at gate node. (a) and (b) Voltage waveforms for $d=0.4 \mathrm{~m}$ and $d=0.35 \mathrm{~m}$, respectively. (c) Measured spectra for $d=0.38 \mathrm{~m}$.

\section{CONCLUSION}

An investigation of wireless coupled oscillator systems for distributed synchronization of local oscillators has been presented. It is based on a realistic description of the oscillator elements and the propagation-dependent coupling effects. The distinct oscillation modes due to the system symmetry have been identified. Very good agreement has been obtained with costly circuit-level simulations and measurements.

\section{ACKNOWLEDGMENT}

The authors would like to thank to Spanish Ministry of Economy and Competitiveness for their financial support under the research project TEC2014-60283-C3-1-R, the European Regional Development Fund (ERDF/FEDER), Juan de la Cierva Research Program IJCI-2014-19141 and the Parliament of Cantabria for financial support under the project Cantabria Explora 12.JP02.64069.

\section{REFERENCES}

[1] O. Simeone, U. Spagnolini, Y. Bar-Ness and S. H. Strogatz, "Distributed synchronization in wireless networks," IEEE Signal Processing Magazine, vol. 25, no. 5, pp. 81-97, Sep., 2008.

[2] O. Simenone, U. Spagnolini, "Distributed synchronization for wireless sensor networks with coupled discrete-time oscillators, Eurasip J. Wireless Commun. Networking, \#57054, 2007.

[3] S. Barbarossa, G. Scutari, "Decentralized maximum likelihood estimation for sensor networks composed of nonlinearly coupled dynamical systems," IEEE Trans. Signal Processing, vol. 55, no. 7, pp. 3456-3470, Jul. 2007.

[4] F. Ramírez, E. de Cos, A. Suárez, "Nonlinear analysis tools for the optimized design of harmonic-injection frequency dividers", IEEE Trans. Microw. Theory Tech., vol. 51, no. 6, pp. 1752-1762, Jun. 2003.

[5] A. Suárez, F. Ramírez, S. Sancho, "Stability and Noise Analysis of Coupled-Oscillator Systems," IEEE Trans. Microw. Theory Tech., vol. 59, no. 4, pp. 1032-1046, Apr., 2011.

[6] R. A. York, "Nonlinear analysis of phase relationships in quasi-optical oscillator arrays," IEEE Trans. Microw. Theory Tech., vol. 41, no. 10, pp. 1799 - 1809, Oct. 1993.

[7] M. Pontón and A. Suárez, "Analysis of self-injection locked oscillators for motion sensing applications," IEEE MTT-S Int. Microwave Symp., San Francisco, CA, May 2016.

[8] K. Kurokawa, "An Analysis of Rucker's Multidevice Symmetrical Oscillator," IEEE Trans. Microw. Theory Techn., vol. 18, pp. 967, May. 1970.

[9] F. Ramírez, M. Pontón, S. Sancho, A. Suárez, "Stability analysis of oscillation modes in quadruple-push and Rucker's oscillators," IEEE Trans. Microw. Theory Techn., vol. 56, no. 11, pp. 2648-2661, Nov., 2008.

[10] A. Suárez, Analysis and Design of Autonomous Microwave Circuits. Hoboken, NJ: Wiley IEEE Pres, 2009.

[11] K. Kurokawa, "Some basic characteristics of broadband negative resistance oscillators," Bell Syst. Tech. J., vol. 48, pp. 1937-1955, Jul.Aug., 1969.

[12] J. Jugo, J. Portilla, A. Anakabe, A. Suárez, and J. M. Collantes, "Closedloop stability analysis of microwave amplifiers," Electron. Lett., vol. 37, no. 4, pp. 226-228, Mar. 2001. 